\title{
Relación entre el personal de enfermería y las gestantes durante el trabajo de parto
}

\author{
Relationship between the nursing personnel and expecting mothers during labor \\ Relação entre o pessoal de enfermagem e as gestantes durante o trabalho de parto \\ Claudia Patrícia Henao-Lopez* \\ Sandra Patrícia Osorio-Galeano** \\ Diego Alejandro Salazar-Blandón***
}

\section{Resumen}

Objetivo: Describir la relación entre el personal de enfermería y las gestantes durante el trabajo de parto, desde la perspectiva de la mujer. Métodos: Estudio descriptivo y transversal. Se incluyó el 92,4\% del total de las gestantes en trabajo de parto atendidas en una institución de salud de la ciudad de Medellín, entre los meses de junio y julio de 2017. Se aplicó un cuestionario ad hoc, diseñado a la luz de las fases de la relación interpersonal enfermera paciente, descritas por Hildegard Peplau, el cual fue sometido a una prueba piloto y concepto de cinco expertos. Se aplicaron pruebas chi-cuadrado y se construyó un modelo de regresión logística. Resultados: Participaron 540 mujeres con edad promedio de 25,6 años (DE 6,0). El 91,9\% valoran como positiva su relación con la enfermera. Se encontró que, a mayor edad de la gestante, mejor comunicación con el personal de enfermería $(\mathrm{X} 2=15,9 \mathrm{p}=0,003)$ y que las madres con parto previo tuvieron una mejor experiencia frente al cuidado $(X 2=4,28 \mathrm{p}=0,038)$. La regresión logística mostró ( $90 \%$ de confianza) que una relación positiva con el personal de enfermería en el parto está relacionada con: la edad $(\mathrm{OR}=0,9, \mathrm{IC}=0,867-0,989)$, aclarar las dudas frente al parto $(\mathrm{OR}=18,0, \mathrm{IC}=6,641-48,938)$, evitar el frío (OR=6,1, IC $=2,347-16,3)$ y la ayuda para tranquilizarse $(\mathrm{OR}=4,1, \mathrm{IC}=1,45-11,714)$. Conclusión: Durante el cuidado de la gestante en trabajo de parto, es importante aclarar las dudas, brindar medidas de confort y generar estrategias para tranquilizarla, así como para establecer una relación interpersonal positiva en la experiencia del trabajo de parto

Palabras clave: Atención de enfermería, trabajo de parto, relación enfermera paciente.

\section{Abstract}

Objective: Describe the relationship between the nursing personnel and the expecting mothers during labor, from the perspective of women. Methods: Descriptive and crosssectional study. From the total expecting mothers, 92,4\% were included during labor and were attended in a healthcare institution in the city of Medellin between the months or June and July, 2017. A type ad hoc questionnaire was applied, designed in light of the phases of the interpersonal relationship between the nurse and patient, described by Hildegard Peplau, which was subject to a pilot experiment and concept of five experts. Chi-
Autor de correspondencia

* Enfermera. Magíster en Enfermería. Docente Universidad Pontificia Bolivariana. Correo: claudia.henao@upb.edu.co. Orcid: https://orcid.org/0000-0001-6139-2712. Medellín, Colombia.

**Enfermera. Especialista en enfermería neonatal, Magíster en epidemiologia. Docente facultad de Enfermería Universidad de Antioquia. Correo: sandra.osorio@udea. edu.co. Orcid: https://Orcid.org/0000-00019868-2035. Medellín, Colombia.

***Matemático. Magíster en Ciencias Estadísticas. Docente Facultad de Enfermería. Universidad de Antioquia.. Correo: alejandro.salazar@udea.edu.co Orcid: https:// orcid.org/0000-0002-8724-7705. Medellín, Colombia 
ISSN-PRINT

1794-9831

E-ISSN 2322-7028

Vol. 17 No. 1

Ene - Abr 2020

Cúcuta, Colombia

squared tests were applied and a model or logistic regression was built. Results: 540 women participated with an average age of 25,6 years (SD 6,0). 91,9\% rate as positive their relationship with the nurse. It was found that the older the expecting mother is, the better the communication with the nursing personnel (X2 $=15,9 \mathrm{p}=0,003)$ and also that mothers with previous births had a better experience of care $(\mathrm{X} 2=4,28$ $\mathrm{p}=0,038)$. The logistic regression showed ( $90 \%$ reliability) that a positive relationship with the nursing personnel during birth is related to: age $(\mathrm{OR}=0,9, \mathrm{IC}=0,867-0,989)$, clarifying questions about giving birth $(\mathrm{OR}=18,0, \mathrm{IC}=6,641-48,938)$, avoid coldness $(\mathrm{OR}=6,1, \mathrm{IC}=2,347-16,3)$ and help the mother to be calm $(\mathrm{OR}=4,1, \mathrm{IC}=1,45-11,714)$. Conclusion: During the care for the birth of the expecting mother, it is important to clarify questions, provide comfort measures and generate strategies to calm the mother, as well as to establish a positive interpersonal relationship during the birth experience

Keywords: Nursing care, labor, nurse patient relationship.

\section{Resumo}

Objetivo: Descrever a relação entre o pessoal de enfermagem e as gestantes durante o trabalho de parto, desde a perspectiva da grávida. Métodos: Estudo descritivo, transversal. Estudaram-se o 92.4\% das gestantes em trabalho de parto atendidas numa instituição de saúde da cidade de Medellín, entre os meses de junho e julho de 2017. Aplicou-se o questionário Ad Hoc, desenhado à luz das fases do relacionamento interpessoal enfermeira-paciente, descritas por Hildegard Peplau, submetido a prova piloto e conceito de cinco expertos. Aplicaram-se provas de Chi-quadrado e construiu-se um modelo de regressão logística. Resultados: Participaram 540 mulheres com idade média de 25.6 anos (DE6.0). O 91.9\% considerou positivo seu relacionamento com a enfermeira. Encontrou-se que a maior idade a gestante comunicase melhor com o pessoal de enfermagem $(\mathrm{X} 2=15,9 \mathrm{p}=0,003)$ e que as gestantes com gravidez previa tiveram melhor experiência frente ao cuidado $(\mathrm{X} 2=4,28 \mathrm{p}=0,038)$. A regressão logística mostrou $(90 \%$ de confiança) que o relacionamento positivo com a enfermeira no trabalho de parto esteve relacionado com as variáveis: idade $(\mathrm{OR}=0,9, \mathrm{IC}=0,867-0,989)$, esclarecer dúvidas do parto $(\mathrm{OR}=18,0, \mathrm{IC}=6,641$ $-48,938)$, evitar a friagem $(\mathrm{OR}=6,1, \mathrm{IC}=2,347-16,3)$ e a ajuda para tranquilizar-se $(\mathrm{OR}=4,1, \mathrm{IC}=$ 1,45 - 11,714). Conclusão: Durante o cuidado da gestante no trabalho de parto, é importante esclarecer dúvidas, prestar medidas de conforto, gerar estratégias para tranquiliza-la, assim como estabelecer um relacionamento interpessoal positivo na experiência do trabalho de parto.

Palavras-chave: Cuidados de enfermagem, trabalho de parto, relação enfermeiro-paciente.

\section{Introducción}

La relación establecida durante el cuidado de las gestantes en el parto es un tema de constante análisis y reflexión para el personal de enfermería. El trabajo de parto es un proceso que implica riesgos biológicos y emocionales, por lo tanto, las mujeres requieren un cuidado de enfermería que las ayude a sentirse seguras y apoyadas para enfrentar el trabajo de parto (1).

Un adecuado y continuo acompañamiento por parte del personal de Enfermería durante la fase de trabajo de parto, es fundamental para la detección precoz de posibles complicaciones, además, contribuye a la satisfacción de la mujer durante la experiencia del parto y al establecimiento del vínculo madre e hijo (2-4). De igual forma, el acompañamiento de una persona elegida por la gestante y del personal de enfermería, así como la relación interpersonal establecida durante el cuidado, han sido descritos como elementos de gran valoración para las gestantes $(5,6)$. Se ha evidenciado que las mujeres que reciben un apoyo continuo durante el trabajo de parto tienen menor probabilidad de requerir cesárea o analgésicos, y atraviesan por un trabajo de parto más corto; además, las madres que tienen un apoyo continuo tienen una experiencia más positiva frente al parto y se sienten más motivadas a la iniciación de la lactancia materna después del nacimiento de su hijo $(2,7,8)$.

El acompañamiento supone que el personal de enfermería entienda que la persona acompañada es la protagonista y quien acompaña debe adaptarse a sus necesidades, demandas, prioridades y valores (9).

El acompañamiento requiere por lo tanto de empatía, saber escuchar y responder a las necesidades del otro (9). Según el diccionario de la Real Academia Española, acompañar también significa "participar en los sentimientos de alguien" (10). Es así como el acompañamiento toma forma dentro de las relaciones interpersonales durante el cuidado. Para Peplau la enfermería es una relación humana entre una persona que necesita de 
los servicios de salud y un personal con formación especializada en responder a las necesidades de ayuda. Es de esta forma que se puede establecer que las relaciones personales son determinantes en la calidad del acompañamiento.

La relación interpersonal durante el cuidado del trabajo de parto es un elemento esencial de la calidad de la atención, pues posibilita no solo el cuidado desde la esfera biológica, sino que permite reconocer la individualidad de cada mujer, sus necesidades y expectativas; además es determinante en su bienestar y su experiencia frente al parto (11-14). Cuando la enfermera centra su atención a partir del respeto por la individualidad de la paciente, ella se sentirá incluida en su proceso de cuidado para el trabajo de parto, y participará de manera activa en el proceso (15-19). Este es un elemento esencial del parto humanizado, que además incluye muchos otros aspectos que tienen que ver con el respeto a la autonomía, la salud y el bienestar de la mujer. El presente estudio centra su interés en las relaciones interpersonales a la luz de la teoría de Hildergad Peplau, con el fin de conocer la relación de cuidado durante el parto y desde esta perspectiva generar aportes al marco general del parto humanizado.

Hildegard Peplau, propone que "la enfermería es, en esencia, un proceso interpersonal” (20). Peplau define cuatro fases mediante las cuales se establecen las relaciones interpersonales durante el cuidado: orientación, identificación, aprovechamiento y resolución. En la fase de orientación se hace énfasis en la expresión de las necesidades y los sentimientos; en la fase de identificación, la enfermera simboliza una persona que ayuda, que proporciona apoyo; en la fase de aprovechamiento, la enfermera ayuda al paciente a utilizar al máximo todas las posibilidades o alternativas de solución que hayan encontrado juntos; $y$, por último, en la fase de resolución se alcanza un nivel de liberación e independencia (20-22). En cada una de las fases es preciso entender el significado de la experiencia para el paciente, a fin de que la enfermera pueda ayudarlo desde la fuerza educativa y terapéutica (22).

Peplau plantea la relación enfermería-paciente, como una manera de construir confianza, y ayudar a las personas en el proceso de enfermedad a identificar sus problemas $(23,24)$. En el caso del cuidado de las mujeres en trabajo de parto, esta relación es un elemento que contribuye a un parto seguro y satisfactorio.

En el establecimiento de la relación interpersonal con las gestantes durante el trabajo de parto intervienen no solo condiciones humanas, sino también factores como: la forma de distribución de trabajo, el alto volumen de pacientes en los servicios de atención, la tecnología, la distribución de roles (25), el tiempo y la experiencia de los cuidadores en el área materno-infantil. Estos elementos plantean la necesidad de avanzar en la comprensión de las relaciones interpersonales durante el cuidado de las gestantes en trabajo de parto.

La descripción de la relación enfermera-paciente, a través de la teoría de Hildegard Peplau, ha permitido orientar el análisis hacia algunos puntos de interés sobre estudios realizados en contextos de cuidado, como áreas de cuidado coronario y en cuidado de la salud mental $(20,26)$. Este trabajo tuvo como objetivo describir la relación establecida entre el personal de enfermería y las gestantes durante el trabajo de parto, desde la perspectiva de las gestantes a la luz de la teoría de H. Peplau.

Los resultados obtenidos orientan hacia estrategias encaminadas a fortalecer y propiciar una relación positiva y eficiente durante el cuidado de enfermería, para responder de forma integral y oportuna a las necesidades de las gestantes durante el proceso de parto.

\section{Objetivos}

\section{Objetivo general}

Describir la relación establecida entre el personal de enfermería y las gestantes durante el trabajo de parto, desde la perspectiva de las mujeres, a la luz de la teoría de $\mathrm{H}$. Peplau.

\section{Objetivos específicos}

- Describir la fase de orientación durante el cuidado de enfermería a la gestante en el trabajo de parto.

- Explorar la fase de identificación en el establecimiento de la relación entre el personal de enfermería y la gestante durante el trabajo parto.

- Describir la fase de aprovechamiento en la relación entre el personal de enfermería y la gestante durante el trabajo de parto.

- Explorar la fase de resolución durante el cuidado de enfermería a la gestante en el trabajo de parto

- Explorar las relaciones existentes entre las variables de estudio. 
ISSN-PRINT

1794-9831

E-ISSN 2322-7028

Vol. 17 No. 1

Ene - Abr 2020

Cúcuta, Colombia

\section{Materiales y Métodos}

Estudio descriptivo transversal, realizado en una unidad de obstetricia de una institución de atención materno infantil de tercer nivel de complejidad de la ciudad de Medellín (Colombia). La población fue de 694 gestantes en trabajo de parto, atendidas en la institución durante los meses de junio y Julio de 2017. De ellas 584 cumplieron con los criterios de inclusión, 18 no aceptaron participar y 26 no fueron incluidas por razones administrativas, como el alta temprana o dificultades para concertar un espacio o momento pertinente para la aplicación del cuestionario. Finalmente, 540 mujeres fueron incluidas en el estudio, lo que corresponde al $92,4 \%$ del total de la población que cumplió con los criterios de inclusión.

Se incluyeron mujeres posparto entre las 18 y 24 horas, cuyo trabajo de parto y parto hubieran sido atendidos en la institución. Se excluyeron a las gestantes menores de 14 años, o que estuvieran en fase expulsiva, mujeres con alteración del estado de conciencia o con discapacidad auditiva o del habla; se excluyeron también a las gestantes con inestabilidad en el estado de salud, mujeres cuyo hijo presentara malformaciones congénitas o muerte y mujeres en posparto que hayan dejado a su recién nacido en la unidad de neonatos por condiciones clínicas.

La recolección de la información se realizó a través de la aplicación de una encuesta diseñada con un propósito descriptivo a las gestantes. Las fases propuestas por $\mathrm{H}$. Peplau, fueron en el presente estudio, una guía para la descripción de la relación enfermera y gestante durante el cuidado del trabajo de parto. Se revisó y se analizó cuidadosamente la definición y los elementos que la autora describe en cada fase. Posteriormente se establecieron variables y se operacionalizaron en preguntas que, de acuerdo con la realidad de la atención de las gestantes, tienen que ver con cada una de las fases. Así, por ejemplo, en la fase de Orientación las preguntas estaban dirigidas a aspectos relacionados con identificación de la enfermera, con la información acerca del proceso del parto y la atención, entre otros aspectos. Esto es coherente con la descripción que Peplau realiza frente a esta fase, donde afirma que el paciente tiene una necesidad percibida y busca ayuda profesional. En esta fase el personal de enfermería le ayuda a comprender su problema (20). Para el caso de la gestante, ella busca ayuda para dar a luz a su hijo e identifica en el personal de enfermería que le oriente en su proceso, para una mejor comprensión de éste.

La encuesta no buscaba medir o valorar el fenómeno, solo describirlo para una mejor comprensión y para generar hipótesis que permitan estudios de alcance analítico.
Siguiendo esta perspectiva se construyó un cuestionario de 47 preguntas dicotómicas y tipo Likert, donde las variables estaban agrupadas en cinco secciones: información general que incluía las variables sociodemográficas (12 preguntas); fase de orientación, que contemplaba variables relacionadas con la información brindada por la enfermera al ingreso de la gestante (13 preguntas); fase de identificación, que consideraba variables relacionadas con la identificación de las necesidades de la gestante por parte de la enfermera y el reconocimiento personal durante la atención (9 preguntas); fase de aprovechamiento, que incluía variables relacionadas con la capacidad de la gestante de reconocer la ayuda que le puede brindar la enfermera y cómo puede aprovechar esta ayuda (5 preguntas); y fase de resolución, que consideraba variables que tienen que ver con: la capacidad de la gestante para actuar por sí misma, con aspectos como la utilidad de la educación brindada, con la experiencia del parto y si fue importante la relación con el personal de enfermería durante el trabajo de parto ( 8 preguntas). La encuesta fue aplicada a las madres durante la estancia hospitalaria entre las 18- 24 horas después del parto, antes del alta.

La encuesta fue revisada por 5 expertos, con el fin de generar apreciaciones y opiniones que permitieron ajustar las variables según la teoría de Peplau y describir, a la luz de ésta, la relación enfermera-paciente durante el trabajo de parto. Se realizó una prueba piloto en la unidad materno-infantil de III nivel de la ciudad de Medellín donde se adelantó el estudio. Para la selección de las participantes se verificó el libro foliado donde son consignados todos los partos atendidos en la institución; se revisó la historia clínica para considerar el cumplimiento de los criterios de inclusión. En total se realizaron 22 encuestas. La prueba piloto permitió establecer el tiempo promedio para llenar la encuesta que fue de 10 a $15 \mathrm{~min}$, el horario y las condiciones más convenientes para el diligenciamiento por parte de las madres, así como el ajuste del cuestionario frente a la claridad y pertinencia de algunas preguntas y opciones de respuesta.

Para procesar los datos, se utilizó el software IBM SPSS 23; se calcularon medidas de tendencia central, dispersión y frecuencias, para el análisis descriptivo. Bajo una perspectiva exploratoria, se aplicó un modelo de regresión logística. Se consideraron las variables que parecían ser influenciadas por otras, para contrastarlas mediante regresión logística. Es así, como se estableció la variable dependiente para efectos del modelo: importancia que tiene para la gestante la relación con el personal de enfermería durante el trabajo de parto. Como variables inde- 
pendientes se seleccionaron aquellas que se sospecharon (por análisis descriptivo y teórico) pudieran influenciar dicha percepción, entre ellas: tiempo en horas de cuidado de enfermería durante el trabajo de parto, edad, reconocimiento de las expectativas y deseos frente al trabajo de parto, si recibió orientaciones acerca de la respiración durante la contracción, indicaciones a la familia acerca de la evolución del trabajo de parto, oportunidad de expresar sentimientos frente a la labor del trabajo de parto, sentirse escuchada cuando hablaba al personal de enfermería, si recibió cuidado del pudor y privacidad durante la realización de los procedimientos, le evitó el frío, sintió confianza y seguridad, recibió ayuda para tranquilizarse durante el trabajo de parto, refuerzos de analgesia para controlar el dolor, si fueron administrados de manera oportuna, aclaración de dudas frente al parto, entre otras. Estas fueron incluidas en un modelo inicial y posteriormente, se fue depurando, eliminando una a una las variables independientes que en las estimaciones de sus coeficientes arrojaran un valor de $p \geq 0.10$, buscando un modelo final que identificara las variables explicativas y optimizara el porcentaje de clasificación correcta.

Se tuvieron en consideración los aspectos éticos contemplados en la Resolución 008430 de 1993, los principios éticos de la Ley 911 de 2004, y las pautas del Comité de Organizaciones de las Ciencias Médicas (CIOMS) (2729). El estudio fue aprobado por el comité de ética de investigación de la institución académica en el acta CEIFE 2017-10 y de la institución de salud donde se realizó el estudio con el acta N 6 del 22 de mayo 2017.

\section{Resultados}

En el estudio participaron 540 gestantes que llevaron a cabo el trabajo de parto en una unidad materno-infantil de III nivel de la ciudad de Medellín. La edad promedio de las mujeres fue de $25 \pm 6,0$ años (mínimo de 14 y máximo de 42). El promedio de la edad gestacional al momento del trabajo de parto fue de $38,3 \pm 1,35$ semanas (mínimo de 34 y máximo 42).

El 16,9\% de las mujeres pertenecían al estrato uno, el $46,2 \%$ al estrato dos y el $32,6 \%$ al estrato tres, el resto pertenecían al estrato cuatro, cinco y seis. En cuanto a la escolaridad: el 0,4 no tenían estudio, el 5,2\% formación primaria, el 49,9\% secundaria y formación técnica, el $43 \%$ universitaria y $1,9 \%$ formación de posgrado. Para el $58 \%$ de las mujeres era el primer parto, mientras que $42 \%$ tenían al menos una experiencia de un parto previo. De estas últimas, el 75,7\% expresaron haber tenido una experiencia previa positiva, el $17,1 \%$ una experiencia regular y el 7,1\% mala.

El 98,1\% de las mujeres asistieron al control prenatal. Frente a la clasificación del riesgo de la gestación, el 44, $1 \%$ fueron de alto riesgo y el $55,9 \%$ de bajo riesgo. $\mathrm{Al}$ indagar acerca de la presencia de alguna enfermedad al momento del parto el $27,8 \%$ del total de las gestantes tuvieron al menos una enfermedad, entre las que se destacaron trastornos hipertensivos del embarazo, diabetes gestacional, diabetes tipo I, II e hipotiroidismo.

También se tuvo en cuenta, que una vez que las madres son ingresadas por el servicio de urgencias se inicia la atención general y el cuidado por parte del personal de enfermería; se identificó que el tiempo promedio de cuidado fue de 5,48 horas (DE 3,4), con un mínimo de 1 hora y un máximo de 24 .

La fase de orientación implica el reconocimiento personal de quien cuida y de quien es cuidado. En este sentido, el personal de enfermería se identificó por su nombre desde el inicio de la atención en un 93, 5\% de los casos. La forma como el personal de enfermería se refería a la gestante en el momento de la atención fue: por su nombre el $68 \%$ de los casos, por el número de la camilla el $8,1 \%$ y de otra manera el 23,9\%. Cuando la gestante fue llamada de otra forma, las palabras utilizadas fueron: mamá en el 68,2\% de los casos, gorda/gordita en el 19,3\% y muñeca el 12,4\%. La interacción entre el personal de enfermería y la paciente supone explicar y orientar previamente las intervenciones de enfermería que serán realizadas durante el cuidado del trabajo de parto; en este sentido el $90 \%$ de las madres casi siempre y siempre manifestaron que les explicaron.

Durante el cuidado del trabajo de parto ocurren una serie de eventos en la gestante que implican algunas intervenciones. Se les preguntó a las madres si fueron informadas acerca de que se les realizarían tactos vaginales cada tres horas para valorar la dilatación, en éste sentido el 64,3\% de las mujeres indicaron que el personal de enfermería siempre les informó y el 22,6\% manifestaron que nunca les advirtieron sobre este procedimiento. Al preguntar si el personal de enfermería les explicó que las contracciones iban a aumentar en frecuencia, intensidad y duración, el 73,3\% de las madres refirieron que siempre lo hicieron y al $12 \%$ no les explicaron sobre la dinámica de las contracciones. Por otra parte, el $67,4 \%$ de las gestantes manifestaron que la enfermera les explicaba cómo debían manejar la respiración cuando se presentaban las contracciones. 
ISSN-PRINT

1794-9831

E-ISSN 2322-7028

Vol. 17 No. 1

Ene - Abr 2020

Cúcuta, Colombia
En la fase de identificación el personal de enfermería debe reconocer al ingreso lo que siente la gestante, esta valoración puede realizarse, por ejemplo, preguntado a la gestante qué siente cuando le informan que se quedará hospitalizada para la atención del parto; al respecto, el $60,3 \%$ dijeron sentirse tranquilas, el $20 \%$ con miedo y del $19,6 \%$ restantes, otros sentimientos como alegría, susto y ansiedad.

En esta fase la enfermera debe permitir que la paciente exprese sus sentimientos. Se encontró que el $68,1 \%$ de las gestantes no lo pudieron hacer. Los sentimientos que transmitió el personal de enfermería al inicio de su tra- bajo de parto fueron: optimismo el $87.5 \%$, desamparo el $5,7 \%$ y otros sentimientos el $6,5 \%$.

Por otra parte, el personal de enfermería debe tener una actitud de escucha a las necesidades de las gestantes ante el problema planteado. Al respecto el $72,8 \%$ de las gestantes se sintieron escuchadas, el $26,5 \%$ a veces y el $0,7 \%$ nunca.

Continuando con la fase de identificación en la Tabla 1 se presentan los resultados acerca de las medidas de confort y comodidad que las madres recibieron por parte del personal de enfermería durante el parto.

Tabla 1. Orientaciones del personal de enfermería durante el trabajo de parto respecto al confort y la comodidad

\begin{tabular}{|c|c|c|c|}
\hline Confort y la comodidad & No. & $\%$ & \\
\hline \multirow[t]{3}{*}{ Cuidó su pudor y privacidad durante la realización de los procedimientos } & Sí & 425 & 78,7 \\
\hline & No & 111 & 20,6 \\
\hline & NA & 4 & 0,7 \\
\hline \multirow[t]{3}{*}{ Atendió a su dolor cuando presentaba la contracción y resolvió su necesidad } & Sí & 479 & 88,7 \\
\hline & No & 51 & 9,4 \\
\hline & NA & 10 & 1,9 \\
\hline \multirow[t]{3}{*}{ Le humedeció los labios y calmó su sed } & Sí & 313 & 58,0 \\
\hline & No & 170 & 31,5 \\
\hline & NA & 57 & 10,6 \\
\hline \multirow[t]{3}{*}{ Le evitó el frío ofreciendo mantas calientes, cobijas } & Sí & 477 & 88,3 \\
\hline & No & 43 & 8,0 \\
\hline & NA & 20 & 3,7 \\
\hline \multirow[t]{3}{*}{ Le ayudó para ir al baño o le facilitó el pato para realizar sus necesidades } & Sí & 429 & 79,4 \\
\hline & No & 53 & 9,8 \\
\hline & NA & 58 & 10,7 \\
\hline
\end{tabular}

Fuente: Relación establecida entre el personal de enfermería y las gestantes durante el trabajo de parto a la luz de la teoría de H. Peplau. NA=No aplica

En la fase de aprovechamiento se encontró que, durante el trabajo de parto el $66,6 \%$ de las gestantes sintieron que el personal de enfermería siempre las ayudó a tranquilizarse, el 16,85\% señaló que casi siempre, el 14,26\% que a veces y el 2,22\% manifestó que nunca. Cuando la paciente solicitó al personal de enfermería: el 65,7\% señaló que siempre la atendieron, el $24,4 \%$ que casi siempre lo hicieron, el 9,35 a veces lo hacían y el $0,6 \%$ dijo que nunca la atendieron.

Acerca de la percepción de los cuidados de enfermería durante el trabajo de parto: el $83,9 \%$ manifestaron que eran adecuados, el 0,6\% inadecuado y el 15,6\% respondieron que pudieron haber sido mejores. Las gestantes se sintieron seguras durante la atención en un $60,2 \%$, tran- quilas el $66,5 \%$, con dudas el $8 \%$, molestas el $5,2 \%$ y solas el $4,4 \%$.

En la fase de resolución el 91\% de las madres indicaron que la información que les proporcionó el personal de enfermería fue útil. Al final de la atención se les preguntó cómo había sido su experiencia sobre el cuidado que recibieron por parte del personal de enfermería; al respecto, el $91,9 \%$ de las mujeres respondió que fue positiva.

Después del proceso del parto, la madre se prepara para el posparto y el cuidado de su hijo. Al final, la paciente evalúa la comunicación con el personal de enfermería, en donde se observa que el $86,7 \%$ de las madres indicó que fue buena, seguido del 13,1 y $0,2 \%$ quienes señalaron que fue regular y mala, respectivamente. 
.

ISSN-PRINT

1794-9831

Finalmente, se les pidió a las madres, definir si la relación personal establecida con la enfermera fue importante en su proceso de trabajo de parto o si no lo fue; el 92,6\% de las mujeres manifestó que la relación que se estableció con la enfermera fue importante en su experiencia de parto.

Tabla 2. Relación entre grupo de edad y comunicación con el personal de enfermería

\begin{tabular}{ccccccccc}
\hline La comunicación con el personal de enfermería fue: & Grupos de edades & \multicolumn{1}{c}{} \\
\hline & $<18$ años & $18-35$ años & $>35$ & $\chi 2$ & $p$ & \\
& No. & $\%$ & No. & $\%$ & No. & $\%$ & \\
Buena & 19 & 76 & 402 & 86,5 & 47 & 94 & 15,9 & 0,003 \\
Regular & 6 & 24 & 63 & 13,5 & 2 & 4 & \\
Mala & 0 & 0 & 0 & 0 & 1 & 2 \\
\hline
\end{tabular}

Fuente: Relación establecida entre el personal de enfermería y las gestantes durante el trabajo de parto a la luz de la teoría de H. Peplau.

Al indagar sobre las posibles relaciones se encontró que a mayor edad existe una mejor comunicación (X2 15,9; $\mathrm{p}=0,003$ ) (Tabla 2). Por otra parte, también se halló correlación entre la experiencia del cuidado durante el parto y el haber tenido partos previos. Las madres que tenían experiencia de un parto previo tuvieron una mejor experiencia frente al cuidado durante el parto $(\mathrm{X} 2=4,28 \mathrm{p}=$ $0,038)$ Tabla 3.

Tabla 3. Relación entre partos previos y variables relacionadas con percepción frente a las características del cuidado del personal de enfermería

\begin{tabular}{|c|c|c|c|c|c|c|c|}
\hline Proceso durante el parto & Ha tenido partos previos & & & & & & \\
\hline & Sí & No & $x^{2}$ & $\mathrm{p}$ & & & \\
\hline \multirow[t]{4}{*}{$\begin{array}{l}\text { El personal de enfermería le ayudó a tranquilizarse } \\
\text { durante el trabajo de parto }\end{array}$} & Siempre & 168 & $46,7 \%$ & 192 & $53,3 \%$ & 12,171 & 0,007 \\
\hline & Casi siempre & 35 & $38,5 \%$ & 56 & $61,5 \%$ & & \\
\hline & A veces & 20 & $26 \%$ & 57 & $74 \%$ & & \\
\hline & Nunca & 4 & $33,3 \%$ & 8 & $66,7 \%$ & & \\
\hline \multirow[t]{2}{*}{$\begin{array}{l}\text { El personal de enfermería fue amable cuando le brindaba } \\
\text { la atención }\end{array}$} & Sí & 217 & $43,7 \%$ & 280 & $56,3 \%$ & 6,763 & 0,009 \\
\hline & No & 10 & $23,3 \%$ & 33 & $76,7 \%$ & & \\
\hline \multirow[t]{2}{*}{$\begin{array}{l}\text { La relación que estableció con el personal de enfermería } \\
\text { fue importante en su experiencia de parto }\end{array}$} & Sí & 218 & $43,6 \%$ & 282 & $56,4 \%$ & 6,767 & 0,009 \\
\hline & No & 9 & $22,5 \%$ & 31 & $77,5 \%$ & & \\
\hline
\end{tabular}

Fuente: Investigación Relación establecida entre el personal de enfermería y las gestantes durante el trabajo de parto a la luz de la teoría de H. Peplau.

Durante la fase de resolución se les pidió a las madres, definir si "la relación personal establecida con la enfermera fue importante en su proceso de trabajo de parto (Si/No)". Se consideró este ítem como una variable importante de desenlace para identificar qué aspectos del cuidado pueden influir en esta percepción, se exploraron modelos de regresión logística multivariada donde las variables independientes eran las demás preguntas de la encuesta que se sospechaba podrían estar relacionadas con la variable dependiente. Se exploraron variables como alivio del dolor, relación con la persona que la acompaña, conocimiento de derechos y de deberes, entre muchas otras que tienen que ver con la relación enfermera-paciente a la luz de la teoría de H, Peplau. Se presentan en la tabla 4, los resultados del modelo de regresión, incluyendo aquellas variables con significancia estadística. 
ISSN-PRINT

1794-9831

E-ISSN 2322-7028

Vol. 17 No. 1

Ene - Abr 2020

Cúcuta, Colombia

Tabla 4. Variables relacionadas con la importancia que tiene para la gestante la relación con el personal de enfermería durante el trabajo de parto

\begin{tabular}{|c|c|c|c|c|c|c|c|c|}
\hline \multicolumn{9}{|l|}{ Variables en la ecuación } \\
\hline & B & Error estándar & Wald & $\mathrm{gl}$ & Sig. & OR & \multicolumn{2}{|c|}{$90 \%$ C.I. para OR } \\
\hline & & & & & & & Inferior & Superior \\
\hline Edad & $-0,077$ & 0,04 & 3,716 & 1 & 0,054 & 0,926 & 0,867 & 0,989 \\
\hline Le evitó el frío ofreciendo mantas calientes, cobijas. & 1,822 & 0,589 & 9,57 & 1 & 0,002 & 6,186 & 2,347 & 16,3 \\
\hline Le ayudó a tranquilizarse durante el trabajo de parto. & 1,416 & 0,635 & 4,974 & 1 & 0,026 & 4,122 & 1,45 & 11,714 \\
\hline Le aclaró sus dudas frente al parto. & 2,892 & 0,607 & 22,688 & 1 & 0,00 & 18,028 & 6,641 & 48,938 \\
\hline Constante & 0,851 & 1,125 & 0,573 & 1 & 0,449 & 2,343 & & \\
\hline
\end{tabular}

Fuente: Relación establecida entre el personal de enfermería y las gestantes durante el trabajo de parto a la luz de la teoría de H. Peplau.

\section{Discusión}

La población estudiada tiene características que coinciden con el perfil de las gestantes a nivel nacional y regional. La media de edad de las gestantes en el presente estudio fue de 25 años (DE 6,0), ubicándolas dentro del rango de mayor tasa de fecundidad informado por el DANE en el año 2016, el cual se sitúa entre los 20 y 29 años (30). En Antioquia esta situación es similar en tanto que el 52,3\% de las gestantes del departamento se ubican en este rango de edad (30). Respecto al estrato socioeconómico de las mujeres que participaron en el estudio predomina el estrato 2. Estos datos guardan coherencia con los datos poblacionales del municipio de Medellín, donde la población también se ubica predominantemente en el estrato 2 (31).

En Colombia el control prenatal está reglamentado (32) y se espera que todas las gestantes asistan a este tipo de controles para la identificación temprana de riesgos y complicaciones. El 98,1\% de las mujeres asistieron al control prenatal, lo cual está por encima de la meta nacional propuesta por el Ministerio de Protección Social, que es del $95 \%$ de cobertura para las mujeres del área urbana (33).

Por otra parte, es importante analizar el panorama de las complicaciones relacionadas con la gestación. Al respecto, en el grupo de estudio, la más común estuvo relacionada con los trastornos hipertensivos. Lo que coincide con las cifras de Instituto Nacional de Salud en 2017, donde los trastornos hipertensivos del embarazo son la principal causa agrupada de morbilidad materna (34).

El promedio de horas de cuidado fue de 5,48 horas (DE $3,4)$. Es importante considerar que el tiempo de interacción durante el trabajo de parto con el personal de enfermería puede ser un aspecto que condiciona y determina la relación interpersonal establecida durante el cuidado.
Al respecto, un estudio realizado en Estados Unidos y Canadá con 6915 mujeres, reportó un promedio de 8,1 horas de cuidado por la enfermera desde el ingreso hasta el parto, la mediana fue de 6,6 horas (35).

También se encontró que la experiencia previa de parto tiene correlación con una mejor experiencia frente al cuidado del parto, lo cual coincide con un estudio realizado en el medio oeste de Nepal con 178 madres en estado posparto, en donde se encontró que ser multípara aumentaba dos veces la probabilidad de estar satisfechas con la atención de parto $(\mathrm{OR}=2.352$; IC $=0,801-6,907)(14)$. Este hallazgo da cuenta que para las mujeres que están atravesando su primer parto pueden tener mayor ansiedad y requieren por parte del personal de enfermería un cuidado más cercano, una mayor información y la comprensión de su inexperiencia frente al proceso, para orientarlas de una manera adecuada que responda a sus necesidades.

La fase de orientación comienza con el primer encuentro entre el personal de enfermería y el paciente (20) según Peplau (21); por tanto, tiene que ver con la identificación de quien cuida y de quien es cuidado. En general las gestantes perciben que el personal de enfermería las orienta de manera efectiva; sin embargo, es necesario fortalecer aspectos como la identificación de la persona y el reconocimiento del otro. Al respecto, en un estudio descriptivo realizado en Colombia en el que participaron 80 gestantes en posparto, y que tenía como objetivo describir elementos de humanización en la atención del parto, reportó que el personal de enfermería se dirigió a las mujeres utilizando otros términos y el 58\% la llamaron "mamita" (16).

Así mismo, otro estudio reportó que las personas eran llamadas de manera despersonalizada a través del número de la cama o por la enfermedad del paciente (36). Es necesario llamar a las personas por su nombre, sin uti- 
lizar otros términos de presunta ternura o con otros que nieguen la existencia humana. Todo inicio de una buena relación durante el cuidado se da desde el saludo y desde la primera interacción (37). Conocer e identificar al otro es un aspecto que genera confianza y seguridad. Al generar confianza y seguridad en las madres, las enfermeras crean un ambiente de trabajo seguro para ambas partes y se fortalece la relación interpersonal (18).

Otro aspecto de importancia en el estudio tuvo que ver con la información y la orientación a las gestantes en el proceso del parto. Se ha encontrado que este aspecto ha sido abordado en otros estudios $(13,15)$ y los resultados reafirman que la información es un elemento de gran importancia dentro de la atención del parto. Entre los aspectos que deben ser informados a la gestante a su ingreso se destacan los relacionados con las características de las contracciones, el dolor, la dilatación y el seguimiento del trabajo de parto.

Una investigación realizada en Ghana (África) a 885 mujeres que tuvieron su parto por vía vaginal, reportó que una de las variables predictoras claves para la satisfacción era la calidad de la información proporcionada sobre la condición y el tratamiento de las mujeres durante el trabajo de parto y el parto $(\mathrm{OR}=9,3857, \mathrm{p}=0,007)$ (13). Por otro lado, un estudio efectuado en África, en el que participaron 921 mujeres en posparto, encontró que la mayoría $(77,5 \%)$ tienen una experiencia positiva frente al cuidado durante el parto y que entre los factores predictores de una buena experiencia, se destaca una información oportuna y suficiente frente al proceso $(\mathrm{OR}=1,44$; IC 95\%: 1,03-2,00) (15). También, otro estudio que siguió longitudinalmente a una cohorte de 652 mujeres posparto en el este de Canadá, también exploró los predictores de una buena experiencia durante el parto, concluyendo que el mayor predictor de una experiencia positiva fue la orientación acerca de los eventos durante el parto, afirmando que el grado de conocimientos de la gestante acerca de los eventos que sucederían durante esta etapa mejorarían su experiencia (beta estimado: 4,2; IC 95\%: 3,5-4,0; p: 0,00) (38). En Latinoamérica no se encontraron estudios cuantitativos de este tema en particular, que permitierán comparar resultados obtenidos; no obstante, desde la perspectiva cualitativa se ha descrito que la relación interpersonal, basada en el diálogo y la información, permite una mejor atención durante el proceso del parto y posibilita un vínculo terapéutico que favorece su recuperación (39).

Es necesario recalcar, que estos hallazgos refuerzan la necesidad de mejorar los procesos de orientación; del mismo modo hay que insistir en la importancia que se le debe dar a la información que la madre debe recibir acerca de las intervenciones propias de la atención durante el trabajo de parto, así como aclarar las dudas de las gestantes acerca del parto; todas estas pautas aparecen como un elemento predictor de una relación importante y significativa con el personal de enfermería durante el parto en los resultados de este estudio. Como lo plantea Peplau, durante la fase de orientación la paciente clarifica su primera impresión global del problema, por tanto, el personal de enfermería actúa como un recurso, transmitiendo información específica y ayudando a que la paciente comprenda sus reacciones a los síntomas que acompañan el proceso de un trabajo de parto (21).

Según la teoría de Peplau, las necesidades educativas en la fase de orientación están dirigidas a que la enfermera amplifique la idea que el paciente tiene de su enfermedad y de las intervenciones que va a recibir por parte el grupo de enfermería, con el fin de que la experiencia pueda ser integrada por el paciente tras desarrollar un aprendizaje significativo (21). En el caso de las gestantes, la orientación posibilita una mejor experiencia acerca del parto y el papel del personal de enfermería es fundamental en este sentido.

Durante la fase de identificación, segunda fase de la relación terapéutica, el personal de enfermería y el paciente se conocen. Un aspecto de importancia en el estudio tiene que ver con la necesidad de fortalecer los espacios para que la gestante exprese sus dudas. Esta condición tiene que ver con una comunicación recíproca y bidireccional, en la que se trascienda lo informativo y se llegue a una verdadera interacción y reconocimiento del otro. En el presente estudio el $85 \%$ de las gestantes dijeron haber podido expresar sus dudas, lo cual indica que es importante fortalecer este aspecto, para que todas las madres puedan expresar sus inquietudes durante el proceso de cuidado del parto.

Conviene subrayar, que el expresar inquietudes es de gran importancia, dado que el $20 \%$ de las gestantes dijeron sentir miedo y $19,6 \%$ manifestaron percibir otros sentimientos, entre los que estaban el susto y la ansiedad. De ahí, que el conocer este tipo de sentimientos tiene sentido en tanto que la ansiedad se asocia con una experiencia negativa del parto. Este hallazgo es de gran interés, porque la intensidad de los sentimientos negativos disminuye cuando el paciente se identifica con las personas que le ayudan a sentirse menos amenazado (40). 
ISSN-PRINT

1794-9831

E-ISSN 2322-7028

Vol. 17 No. 1

Ene - Abr 2020

Cúcuta, Colombia
Igualmente, se observó que la relación de ayuda durante la fase de aprovechamiento surge como un aspecto de gran interés, pues el cuidado de enfermería durante el parto está orientado a ayudar a la gestante durante dicho proceso (19). Globalmente, los resultados de la presente investigación son positivos e indican que se genera una respuesta frente a las necesidades de la gestante; no obstante, es necesario promover una respuesta efectiva y positiva en todos los casos. En un estudio realizado en Perú sobre la percepción del paciente frente a la atención indicó que las enfermeras, ante alguna necesidad de cuidado acuden de manera inmediata a brindar la atención: siempre (28\%), a veces (60\%), nunca (12\%) (41). Por tanto, una función del personal de enfermería es captar las necesidades del otro, con el fin de ayudarle a confrontar, aceptar y hacer frente a la situación actual (42).

También, se encontró que en la fase de resolución la gestante elabora una idea acerca de la experiencia global de la relación establecida durante el cuidado del trabajo de parto y determina su importancia dentro de la experiencia general. Al preguntarle si la relación que estableció con el personal de enfermería fue importante en su experiencia de parto, el 92,6\% respondió que sí. Estos resultados validan la importancia de los componentes de las relaciones humanas como un indicador de cuidado. De igual forma, le dan sentido a la importancia del cuidado humanizado frente al cual Jean Watson afirma que el cuidado de la enfermera es un fenómeno social universal, que resulta efectivo si se da esa relación interpersonal en la que el enfermo puede llegar a tener una mejor visión de la situación que vive; esto implica empatía, afecto y comunicación eficaz $(43,44)$.

A largo plazo, las experiencias del parto pueden tener efectos positivos o negativos sobre la vida, el bienestar y salud. Una experiencia positiva puede ser recordada como un evento de vida junto a un crecimiento personal, frente a una experiencia de parto negativa que aumenta el riesgo de resultados de salud adversos, como la depresión posparto y temor futuro de dar a luz, lo que puede conducir a una solicitud de cesárea en futuros embarazos (35).

Hay que mencionar además, que fue posible establecer que las variables que predicen una percepción importante y significativa de la relación con la enfermera durante el parto fueron: la edad, aclarar las dudas frente al parto, ayuda para tranquilizarse durante el trabajo de parto y evitar el frío ofreciendo mantas calientes. En cuanto a la edad, además de aparecer en el modelo de regresión, se encontró que existe una correlación la cual indica que a mayor edad de la gestante en trabajo de parto, la comunicación con el personal de enfermería tiende a ser mejor. Este hallazgo pone de manifiesto la importancia de considerar que las adolescentes tienen una necesidad mayor de atención y por tanto, tienen más dificultades para establecer una comunicación efectiva con el personal de enfermería durante el parto $(45,46)$. Un estudio exploratorio-descriptivo realizado en Brasil (47), cuyo objetivo fue describir los significados atribuidos por las adolescentes a la atención recibida al momento del parto por el personal de enfermería, reporta que solo el $40 \%$ dijeron haber recibido explicación sobre los procedimientos que les iban a realizar. Este hallazgo indica que las adolescentes en general pueden tener una percepción negativa de la información brindada, lo que podría relacionarse con los hallazgos del presente estudio (46).

En el modelo de regresión, la variable "aclarar las dudas acerca del parto" fue la variable que influyó en mayor medida (OR=18,0, IC =6,641 - 48,938.) sobre la percepción de la gestante acerca de la importancia sobre la relación establecida con el personal de enfermería durante la experiencia del parto. Al respecto, la literatura señala que una adecuada información a las mujeres durante su labor de trabajo de parto puede aumentar su nivel de control frente al proceso (48). Además, se indica que la información a las gestantes es determinante a la hora de satisfacer a las madres para que perciban que son escuchadas en sus preocupaciones y para que les aclaren las dudas durante este proceso (48).

Respecto, a quitar el frío y cubrir con mantas calientes y cobijas es la segunda variable de mayor importancia en el modelo de regresión del presente estudio ( $\mathrm{OR}=6,1$, IC $=2,347-16,3)$; razón por la cual, la Doctora Katharine Kolcaba (49) asegura que la mayoría de las incomodidades que experimenta un paciente en el entorno quirúrgico, tienen que ver con el dolor y la hipotermia, y estas a su vez pueden generar sensaciones corporales de disconfort. En este sentido, el personal de enfermería debe orientar intervenciones de confort en el ambiente del cuidado de las gestantes, en quienes este tipo de medidas son de gran importancia. De acuerdo con los resultados del presente estudio, el cuidado orientado a evitar el frío toma especial relevancia, en cuanto a la experiencia del parto y el componente relacional del cuidado.

En el caso del cuidado de las gestantes es necesario considerar que atraviesan por un proceso en el que requieren de apoyo y ayuda constante. Tal y como se ha venido mencionando, una relación positiva permite identificar 
necesidades de confort y en esta medida generar estrategias de cuidado orientadas al mismo. De esta forma se puede precisar que en el cuidado de las gestantes, la aplicación de las teorías de mediano rango, en este caso la teoría del confort, aporta a un cuidado más comprensivo.

Por último, ayudar a tranquilizarse fue otra de las variables relacionada con la importancia de la relación interpersonal de la gestante con el personal de enfermería $(\mathrm{OR}=4,1$, $\mathrm{IC}=1,45-11,714)$. No se encontraron estudios que exploren este aspecto en particular. Pero sí se ha indagado acerca de los factores predictivos de una experiencia positiva frente al parto. Un estudio realizado en África (15) determinó que un factor predictivo de una buena experiencia del parto es ser tratado con respeto y dignidad (OR $1,69=$ IC $95 \%: 1,18-2,43$ ), actitudes que tienen que ver con la posibilidad de expresar sus emociones, participar de las decisiones, manifestar miedos y temores y alcanzar tranquilidad durante el proceso de parto.

Los resultados del presente estudio evidencian que el personal de enfermería puede mejorar la experiencia del parto, a través de estrategias concretas que tienen que ver con las relaciones interpersonales durante el cuidado.

Es necesario recalcar que la importancia del análisis por fases radica en la posibilidad de detallar los elementos que permitieron una construcción global de la experiencia. Las fases descritas por Peplau continúan vigentes y su descripción de la relación enfermería-paciente se presenta como una manera de construir confianza, de ayudar a las personas en el proceso de la enfermedad a identificar sus problemas (fase de orientación) y a trabajarlos (fase de identificación); de igual forma hay que maximizar y aprovechar la prestación de atención física, la enseñanza sobre la salud y el asesoramiento (fase de aprovechamiento), para finalmente, proporcionar el cierre de un trabajo en conjunto (fase de resolución).

Las fases descritas por Peplau, utilizadas como una guía para la descripción de la relación establecida entre las gestantes y el personal de enfermería durante el trabajo de parto, permitieron una mejor comprensión de las necesidades de las gestantes y de la forma como el personal de enfermería puede dar respuesta de la relación interpersonal.

\section{Conclusiones}

La fase de orientación se caracteriza por ser un proceso en el que existe, en general, una adecuada información, la cual se da de manera clara y comprensible. Sin embargo, se identificó que es necesario fortalecer el reconocimiento de la gestante en su individualidad, mediante acciones concretas como identificarla por su nombre, así como la escucha activa de sus expectativas y deseos frente al parto. La paciente necesita información y toda la ayuda para comprender y planificar el uso de los servicios que pueda prestarle el personal de enfermería.

En la fase de identificación, que implica la participación del personal de enfermería para ayudar a la gestante a satisfacer sus necesidades, la percepción de éstas es predominantemente positiva. Las madres identificaron que el personal de enfermería estaba interesado en ayudarles con sus necesidades y en ofrecerles medidas de confort y comodidad durante el trabajo de parto. En esta fase también se identificó que, en general, las madres experimentan diversos sentimientos y que un desafío importante para el personal de enfermería es lograr comprenderlos, para lo cual es necesario indagar acerca de lo que está experimentando la madre en el momento del parto.

Durante la fase de aprovechamiento las gestantes en su mayoría sintieron confianza y seguridad en el personal de enfermería. Además, describen de manera positiva la actitud frente al llamado y a la solicitud de ayuda al personal de enfermería. Sin embargo, en esta fase se hace evidente la necesidad de escuchar a la gestante frente a sus necesidades, deseos y expectativas.

En la fase de resolución las gestantes valoran como útil la información dada y satisfactorio el cuidado recibido durante el proceso del parto. En esta fase es importante fortalecer la comunicación con la familia de la gestante para generar una mejor relación durante el cuidado y mejorar la experiencia del parto.

Las gestantes que tienen una experiencia previa frente al parto y que tienen mayor edad, se relacionan mejor con el personal de enfermería y experimentan una mejor experiencia frente al proceso. Así mismo, reconocen que la relación que se establece con el personal de enfermería es importante durante el trabajo de parto y se ve influenciada por una adecuada información, la ayuda para tranquilizarse y la resolución de sus necesidades frente al confort y la comodidad.

\section{Conflicto de Intereses}

Los autores declaran no tener ningún conflicto de interés 
ISSN-PRINT

$1794-9831$

E-ISSN 2322-7028

Vol. 17 No. 1

Ene - Abr 2020

Cúcuta, Colombia

\section{Referencias Bibliográficas}

1. Organización Mundial de la Salud, UNICEF, UNFPA, Banco Mundial, Naciones Unidas. Evolución de la mortalidad materna: 1990-2015: estimaciones de la OMS, el UNICEF, el UNFPA, el Grupo del Banco Mundial y la División de Población de las Naciones Unidas [Internet]. Santiago de Chile: CEPAL; 2016 [citado 8 de diciembre de 2015]. Disponible en: http://www.who.int/reproductivehealth/ publications/monitoring/maternal-mortality-2015/es/

2. Hodnett ED, Gates S, Hofmeyr GJ, Sakala C. Continuous support for women during childbirth. Cochrane Database Syst Rev [Internet]. 2012;(10). Disponible en: https://www.ncbi.nlm.nih.gov/ pubmed/23857334

3. Acosta S, Bejarano Beltrán N, Bejarano Beltrán M, Castiblanco N, Castañeda L, Castañeda P. Guía de cuidado de enfermería en control prenatal: enfermería basada en la evidencia (EBE) [Internet]. Bogotá: Secretaría Distrital de Salud; 2016 [citado 6 de diciembre de 2016]. Disponible en: http://www. saludcapital.gov.co/DDS/Guas $\% 20 \mathrm{de} \% 20$ cuidado $\% 20 \mathrm{de} \% 20$ enfermera/Guia $\% 20$ prenatal.pdf

4. Cy Chan Z, Wong KS, Lam WM, Wong KY, Kwok YC. An exploration of postpartum women's perspective on desired obstetric nursing qualities. J Clin Nurs. 2014; 23(1-2):103-12.

5. Giraldo Montoya DI, González M, Henao C. Experiencias de las mujeres durante el trabajo de parto y parto. Av En Enferm. 2015; 33(2):271-281.

6. Saavedra D. Comportamientos de cuidado del profesional de enfermería que percibe la gestante en trabajo de parto y parto de una clínica privada. [Tesis Maestría en Enfermería] Bogotá: Universidad Nacional de Colombia; 2013. [citado 11 de diciembre de 2018] Disponible en: http://www.bdigital. unal.edu.co/11168/1/deisylorenasaavedrainfante.2013.pdf

7. Karlström A, Nystedt A, Hildingsson I. The meaning of a very positive birth experience: focus groups discussions with women. BMC Pregnancy Childbirth. 2015 Oct 9;15:251.

8. Bohren MA, Hofmeyr GJ, Sakala C, Fukuzawa RK, Cuthbert A. Continuous support for women during childbirth. Cochrane Database Syst Rev. 2017; 7:CD003766.

9. Sanz P, Jurado M, Fernández M. La humanización de los cuidados, la clave de la satisfacción asistencial: hacia el plan de acogida y acompañamiento [Tesis de maestría] España: Escuela universitaria de enfermería Santa Madrona 2009 [citado 6 de noviembre de 2019] Disponible en http://diposit.ub.edu/ dspace/bitstream/2445/21526/1/2009_2.pdf

10. Diccionario de la Real Academia Española. Concepto de acompañar. [Internet]. [citado 6 de noviembre de 2019] Disponible en: http://dle.rae.es/?id=0XSNNQU

11. Carlsson IM, Ziegert K, Nissen E. The relationship between childbirth self-efficacy and aspects of well-being, birth interventions and birth outcomes. Midwifery. 2015 Oct;31(10):1000-7.

12. Hildingsson I, Haines H, Cross M, Pallant JF, Rubertsson C. Women's satisfaction with antenatal care: Comparing women in Sweden and Australia. Women Birth. 2013;26(1): e9-e14.

13. Avortri GS, Beke A, Abekah-Nkrumah G. Predictors of satisfaction with child birth services in public hospitals in Ghana. Int J Health Care Qual Assur. 2011; 24(3):223-37.

14. Panth Asha, Praveena Kafle. Maternal Satisfaction on Delivery Service among Postnatal Mothers in a Government Hospital, Mid-Western Nepal. Obstetrics and gynecology international. 2018. 11 pages, https://doi.org/10.1155/2018/4530161

15. Mukamurigo JU, Berg M, Ntaganira J, Nyirazinyoye L, Dencker A. Associations between perceptions of care and women's childbirth experience: a population-based cross-sectional study in Rwanda. BMC Pregnancy Childbirth. 2017;17(1):181.

16. Coral Ibarra R. Humanización del cuidado de enfermería durante el parto. Rev UDCA Actual Divulg Científica. 2008;11(1):47-56.

17. Isbir GG, Serçekus P. The Effects of intrapartum supportive care on fear of delivery and labor out- 
comes: A single-blind randomized controlled trial. J Nurs Res. 2017;25(2):112-119.

18. Nikula P, Laukkala H, Pölkki T. Mothers' perceptions of labor support. MCN Am J Matern Child Nurs. Diciembre de 2015; 40(6):373-80.

19. Macpherson I, Roqué-Sánchez MV, Legget FO, Fuertes F, Segarra I. A systematic review of the relationship factor between women and health professionals within the multivariant analysis of maternal satisfaction. Midwifery. 2016; 41:68-78.

20. D'antonio P, Beeber L, Sills G, Naegle M. The future in the past: Hildegard Peplau and interpersonal relations in nursing. Nurs Inq. 2014; 21(4):311-317.

21. Peplau HE. Interpersonal relations in nursing: a conceptual frame of reference for psychodynamic nursing. New York: Springer; 2002.

22. Fawcett J. Contemporary nursing knowledge: Analysis and evaluation of nursing models and theories. 3. a ed. Boston: University of Massachusetts; 2012.

23. Washington GT. The theory of interpersonal relations applied to the preceptor-new graduate relationship. J Nurses. 2013;29(1):24-29.

24. Peterson SJ, Bredow TS. Middle range theories: application to nursing research. 3.a ed. Philadelphia: Lippincott Williams \& Wilkins; 2013.

25. Ceballos Vásquez PA. Desde los ámbitos de enfermería, analizando el cuidado humanizado. Cienc. enferm. Concepcion 2010; 16(1): 31-35.

26. Zarea K, Maghsoudi S, Dashtebozorgi B, Hghighizadeh MH, Javadi M. The impact of Peplau's therapeutic communication model on anxiety and depression in patient's candidate for coronary artery bypass. Clin Pract Epidemiol Ment Health. 2014; 10:159-65.

27. Colombia. Ministerio de Salud y Protección Social. Ley 911 de 2004 por la cual se dictan disposiciones en materia de responsabilidad deontológica para el ejercicio de la profesión de Enfermería en Colombia; se establece el régimen disciplinario correspondiente y se dictan otras disposiciones. Diario Oficial. (45693, Oct 6, 2004).

28. Emanuel E. ¿Qué hace que la investigación clínica sea ética? ¿Siete requisitos éticos? En: Pellegrini Filho A, Macklin R Investigación en sujetos humanos: experiencia internacional. Santiago de Chile: OPS: OMS; 1999. p. 33-46.

29. Consejo de Organizaciones Internacionales de las Ciencias Médicas, Organización Mundial de la Salud. Pautas éticas internacionales para la investigación biomédica en seres humanos [Internet]. Santiago de Chile: CIOMS; 2002 [citado 8 de diciembre de 2016]. Disponible en: http://www.cioms.ch/ publications/guidelines/pautas eticas internacionales.htm

30. Departamento Administrativo Nacional de Estadística. DANE: información estratégica [Internet]. Bogotá: DANE; 2016 [citado 19 de diciembre de 2016]. Disponible en: http://www.dane.gov.co/

31. Encuesta de calidad Medellín: 2015-2016 [Internet]. Tableau; 2016 [citado 19 de diciembre de 2016]. Disponible en: https://public.tableau.com/views/ECV2015-2016Mapav2/Story1?:embed=y\&:display_ count=yes\&:showVizHome $=$ no\#3

32. Colombia. Ministerio de Salud y Protección Social, Colciencias. Guías de práctica clínica para la prevención, detección temprana y tratamiento de las complicaciones del embarazo, parto o puerperio, Guías No. 11-15 [Internet]. Bogotá: Ministerio de Salud y Protección Social: Colciencias; 2013 [citado 4 de diciembre de 2016]. Disponible en: https://www.minsalud.gov.co/sites/rid/Lists/BibliotecaDigital/RIDE/INEC/IETS/Gu\%C3\%ADa.completa.Embarazo.Parto.2013.pdf\#search=\%252Cguia $\% 2520$ de $\% 2520$ trabajo $\% 2520 \mathrm{de} \% 2520$ parto.

33. Profamilia. Encuesta Nacional de Demografía y Salud, componente de salud sexual y salud reproductiva [Internet]. Bogotá: Profamilia; 2015 [citado 19 de diciembre de 2016]. Disponible en: http:// profamilia.org.co/docs/ENDS\%20TOMO\%20II.pdf. 
ISSN-PRINT

1794-9831

E-ISSN 2322-7028

Vol. 17 No. 1

Ene - Abr 2020

Cúcuta, Colombia

34. Narváez Díaz N. Informe del evento morbilidad materna extrema, hasta el periodo epidemiológico 09 del año 2017 [Internet]. Bogotá: Instituto Nacional de Salud, Dirección de Vigilancia y Análisis del Riesgo en Salud Pública; 2017 [citado 14 de febrero de 2017]. Disponible en: http://simposiovirologia. ins.gov.co/lineas-de-accion/Subdireccion-Vigilancia/Informe $\% 20 \mathrm{de} \% 20$ Evento $\% 20$ Epidemiolgico/ MORBILIDAD\%20MATERNA\%20EXTREMA\%20PE\%20IX\%202017.pdf.

35. Hodnett ED, Lowe NK, Hannah ME, Willan AR, Stevens B, Weston JA, et al. Nursing Supportive Care in Labor Trial Group: Effectiveness of nurses as providers of birth labor support in North American hospitals: a randomized controlled trial. JAMA. 2002; 288(11):1373-1381.

36. Prieto G. Humanización del cuidado de enfermería en ética y enfermería. Boletín Latinoamericano. 2000;(4):1-7.

37. Bramhall E. Effective communication skills in nursing practice. Nurs Stand. 2014 Dec 9;29(14):53-9.

38. Bryanton J, Gagnon AJ, Johnston C, Hatem M. Predictors of women's perceptions of the childbirth experience. J Obstet Gynecol Neonatal Nurs. 2008;37(1):24-34.

39. Araújo Rocha FA, Carvalho Fontenele FM, Rodrigues de Carvalho I, Verdes Rodríguez DC, Araújo de Sousa R, Rodrigues Ferreira A. Cuidado no parto e nascimento: percepção de puérperas. Rev Rene. 2015 16(6), 782-789.

40. Bell AF, Andersson E. The birth experience and women's postnatal depression: A systematic review. Midwifery. 2016 Aug; 39:112-23.

41. Silva Fhon J, Ramón-Cordova S, Vergaray-Villanueva S, Palacios FV, Partezani-Rodrigues R. Percepción del paciente hospitalizado respecto a la atención de enfermería en un hospital público. Enferm Univ. 2015;12(2):80-87.

42. Lapeña YR, Cibanal Juan L, Pedraz Marcos A, Macía Soler ML. Interpersonal relationships among hospital nurses and the use of communication skills. Texto contexto - enferm. 2014; 23 (3): 555-562.

43. Watson J, Brewer BB. Caring science research: criteria, evidence, admeasurements. J Nurs Adm. 2015;45(5):235-6.

44. Lenis-Victoria CA, Manrique-Abril FG. Calidad del cuidado de enfermería percibida por pacientes hospitalizados. Aquichan. 2015;15(3):413-425.

45. Donoso E, Carvajal JA, Vera C, Poblete JA. La edad de la mujer como factor de riesgo de mortalidad materna, fetal, neonatal e infantil. Rev Médica Chile. 2014;142(2):168-174.

46. Angarita Gómez JA, Basto Montero M, Noreña Acevedo I, Pardo MP. Expectations of pregnant teenagers about nursing care in labor rooms. Rev cubana Enfermer. 2014; 30(2).

47. Andrade Sampaio Á, Vasconcelos Silva ÂR de, Rejane Ferreira Moura E. Atención humanizada del parto de adolescentes? 'norma, deseo o realidad? Rev Chil Obstet Ginecol. 2008;73(3):185-191.

48. Iravani M, Zarean E, Janghorbani M, Bahrami M. Women's needs and expectations during normal labor and delivery. J Educ Health Promot. 2015; 23; 4:6.

49. Seyedfatemi N, Rafii F, Rezaei M, Kolcaba K. Comfort and hope in the preanesthesia stage in patients undergoing surgery. J Perianesth Nurs. 2014 Jun;29(3):213-20. 